\title{
Short-term Load Forecasting Based On Geographic Information System
}

\section{Tong Li}

\author{
Department of Electrical and Electronic Engineering, North China Electric Power University, \\ Baoding071000, Hebei Province,China;
}

Email:531875450@qq.com

\begin{abstract}
Keywords: Short-term load forecasting,Genetic Annealing Algorithm,Support Vector Machine
\end{abstract}
\begin{abstract}
Short-term load forecasting is inevitable and important for electric power management.In order to predict precisely and reliably, the forecasting model is established combining Genetic Annealing Algorithm and Support Vector Machine, which respectively improve partly search capability and possesses more adaptability.

Then both of them are gathered together to cooperate with Geographic Information System, which can perfectly combine graphic information and attribute data. Design relevant database and platform, then the practical forecasting method for short-term load is complete.
\end{abstract}

\section{Introduction}

Electric power load refers to the total power of all the electric equipment in the power system, which is called load also. What's more, in electric power system, power quality has a close link with power balance. That's to say, it's the unbalance power that will exceedingly effect the safety of electric operation.

With the demand of electric power growing exponentially on the behalf of fast-develop economy, for distribution network, the management is more complex than it ever be, which in turn, will require better management from electric management apartment.

Geographic Information System(GIS) is an especially important spatial information system, which combines both computer hardware and software to collect, store, operate, analyze, depict and describe every aspects of geographic spatial distribution for epigeosphere (including atmosphere). At first, its work field was limited in engineer management and planning department. In the early 90s of 20 centuries, foreign electric companies began to implement AM/FM/GIS (Automated Mapping/ Facilities Management/ Geographic Information System).

\section{Short-term load forecasting in GIS}

The traditional ways for Short-term load forecasting are limited. Time series analysis method, AutoRegressive Moving Average(ARMA ), Seasonal ARIMA, both of these methods are hard to be practical for several specific conditions.

For now, it is normal to develop spatial power forecast system in electric GIS platform and apply GIS ' s marvelous management for spatial data and attribute data, along with its super ability for drawing to accomplish spatial load forecast display.

As a matter of fact, there are not too much research for short-term load forecast in GIS for now. But, many are on-going. In fact, the main difference for these two kinds of forecasting is the thematic map.

Traditional way for result display and analysis is using tables and curve graphs, which is not practical. So it is better to apply graphic analysis, which is also adopted by more and more scholars.

\subsection{Short-term load forecasting overview}

Load forecasting aims at forecasting load value in some future time considering several factors , like the operation characters, politics, economy and climate. The value must meet some precision. It is the foundation to make electric system operating economically and reliably. 


\subsection{Load forecasting feature}

Uncertainty. For electric load, there are too much factors which will effect its forecasting results. Some of them are even more complex. Everything is changeable. In fact, some unpredictable factors also work in this forecasting.

Timeliness. The independent variable is time in this forecasting. Also, for a science forecast like load forecast, time and number of forecasting must be clear.

Multiple solutions. There are several forecasting solutions for each kind of condition.

Conditionity. For the uncertainty of load change, it is essential to have specific assumption and condition for load forecasting. According to historical data, combining with every possible condition, making appropriate assumptions for prediction is necessary.

\section{Short-term load forecasting of SVM based on preferences}

Support Vector Machine(SVM) ${ }^{[1,2]}$ is proposed by Corinna Cortes and Vapnik in 1995, which has an advantage of small sample, nonlinear problem and high dimensional pattern recognition. What's more, it can expand to other machine learning problems like function fitting.

\subsection{SVM regression algorithm}

Establish a sample set .

Where $\mathrm{x}_{\mathrm{i}}$ is input variable and $\mathrm{y}_{\mathrm{i}}$ is output variable.

Train the SVM through this sample set to obtain a regression function $\mathrm{f}(\mathrm{x})$, which is :

- For every input, the precision of outcome must be greater than 1- $\varepsilon$.

- The regression function must be smooth.

As a matter of fact, in terms of network structure, SVM is similar with neural network.

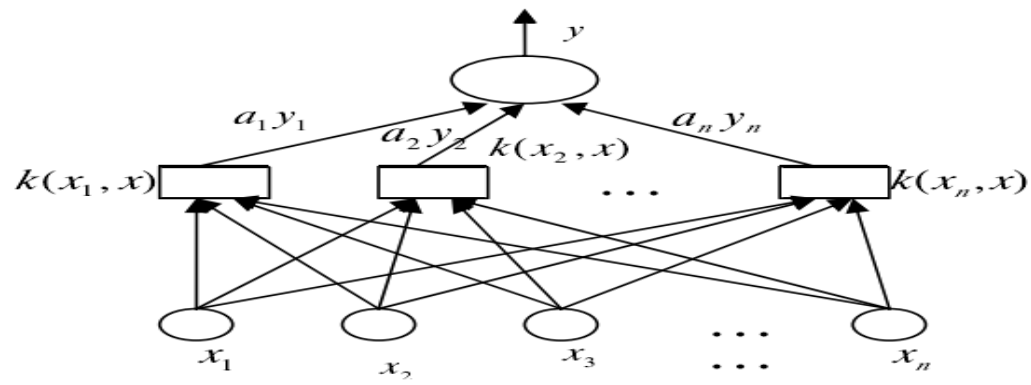

Fig.1 Network Structure of SVM

Figure 1 can describe the process clearly.

\subsection{Characteristic selection of the sample}

From the analysis above, forecasting result is the output of $f(x)$, whose input is the vector composed by its supportive attribute. The elements include historical load,economy,etc. Considering the different influence from different factors, we design the supportive attribute as:

- Daily peak load among 3-days before that day and peak load of that day in last week $L=\left(l_{1}, l_{2}, l_{3}, l\right)$;

- Daily average temperature T.

- Week attribute $W=(1,2,3,4,5,6,7)$;

- Festival attribute $F=(0,1)$, where 1 refers to important festival.

Then, the supportive attribute is $\left\{F, T, W, l_{1}, l_{2}, l_{3}, l\right\}$.

\subsection{Genetic Annealing Algorithm}

Genetic Annealing Algorithm(GAA) ${ }^{[3]}$ is the combination of Genetic Algorithm(GA) and Simulated Annealing Algorithm(SAA). In other words, adding SAA into GA ensure the diversity of solution space and improve partly search capability. In that way, GAA overcome shortcomings and can find a better optimal solution.

\subsection{Optimizing SVM parameters based on GAA}

Then we use GAA to automatically find optimal SVM parameter for sample. 


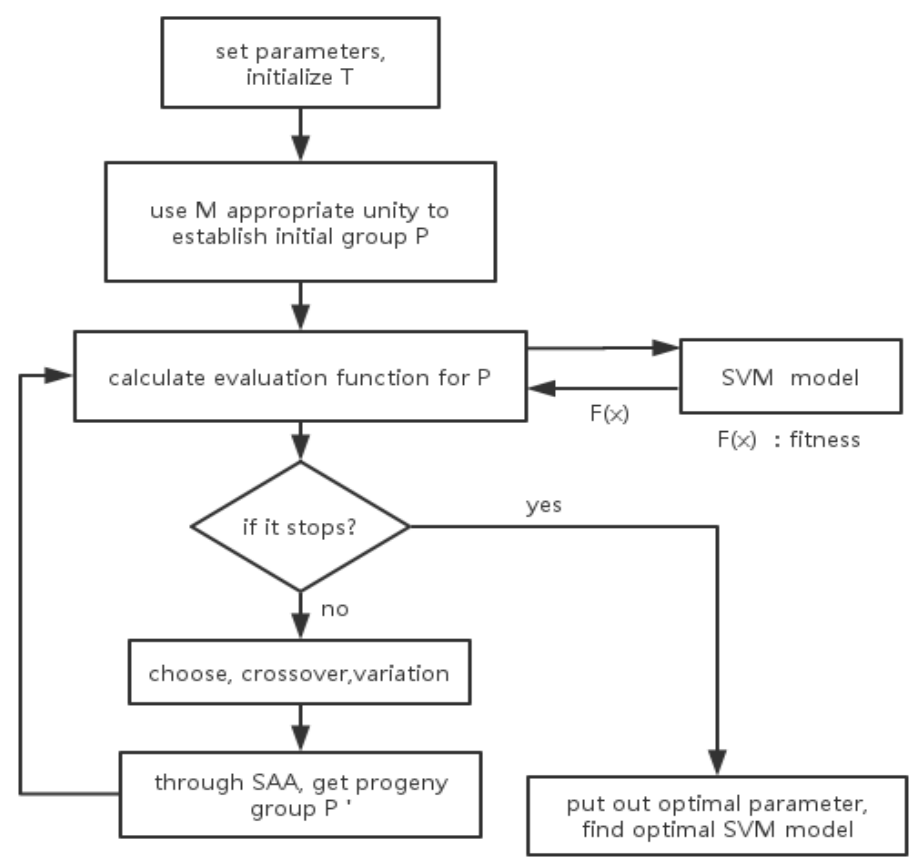

Fig.2 Operation flow chart of GAA-SVM.

Here is the operation flow chart of GAA-SVM.

When it comes to load forecasting, we handle it through:

- Initially process original load sample and normalize training data and test data.

- Establish objective function through training sample.

- Use GAA to find a solution and clarify SVM forecasting model.

- Obtain forecasting value combining supportive attribute of load and SVM model.

Set parameters in SVM as:

$C \in[0.1,5000], \varepsilon \in[0.001,10], \delta \in[0.01,500], \mathrm{M}=100$, hybrid rate is 0.8 , variation rate for individual is 0.1 , annealing rate is 0.96 .

Obtain load data of Baoding power substation and get the forecasting results through above methods. Data from 2015-05-10 to 2015-05-20 is training sample, 2015-05-21 to 2015-05-30 is test sample. Then predict the data from 2015-06-01 to 2015-06-07.Here is the result.

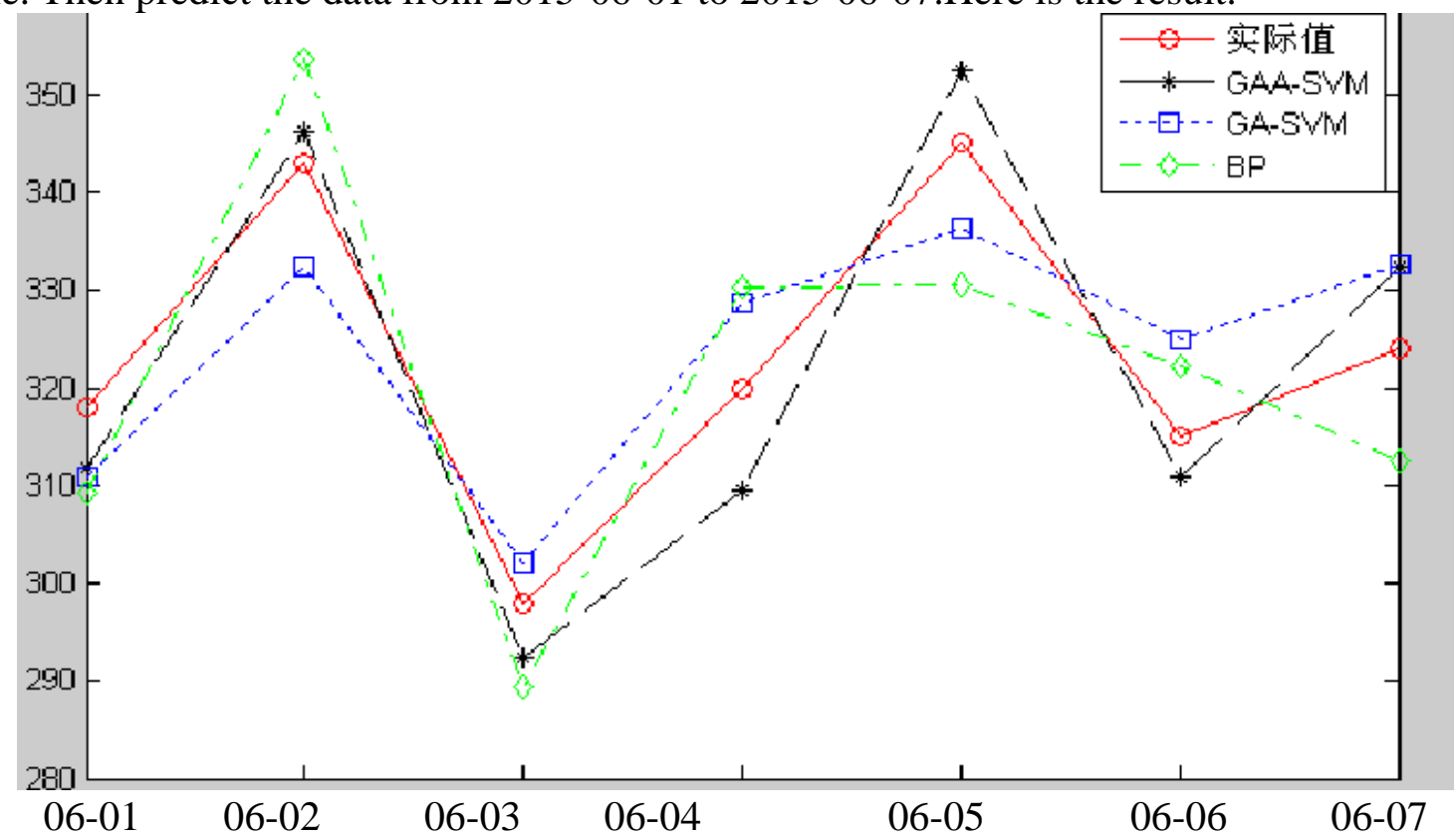

Fig.3 Comparison chart of forecasting result

Through above comparison chart, it is clear that SVM forecasting is more precise than traditional BP neural network algorithm. 


\section{Short-term load forecasting system based on GIS}

The system is based on GIS information management system of Baoding. Arcgis Engine platform is applied also, which can provide diverse rendering interface to design thematic map of load forecasting ${ }^{[4]}$.

As for the design of database, SQL 2000 is used in this system. As the GIS channel between ArcGIS and relative database, ArcSDE is linked with SQL2000. Relevant field include date, date type, average load,total load, maximum load and minimum load, etc.

\section{Conclusion}

Based on the contents above, conclusions can be drawn as follow:

- Through SVM and GAA, short-term load forecasting result is more accurate than that under traditional method like BP neural network method.

- Combining GIS with above forecasting method and design relevant database and platform, an effective system with ability of prediction,result display and analysis for short-term load forecasting can be established.

\section{References}

[1] Bo-Juen Chen,Mingwei Chang, and Chih-Jen Lin. Load Forecasting Using SupportVector Machines: A Study on EUNITE Competition 2001[J]. IEEE TRANSACTION ON POWER SYSTEMS, VOL.19, NO.4, November 2004.

[2] C.-C.Chang and C.-J.Lin.(2001)LIBSVM: A Library for Support Vector Machines.[Online]. Available:http://www.csie.ntu.edu.tw/cjlin/libsvm

[3] Belew R,Booker L. Proceeding of the 4th International Conference on Genetic Algorithms[M]. Los, Altos, CA: Morgan Kaufman Publishers, 1991.

[4]Li Linna: Research \& Development of Short-term load forecasting on Distribution Network Based on GIS, Huazhong University of Science \& Technology 